\title{
Curve Warning Driver Support Systems. A Sensitivity Analysis to Errors in the Estimation of Car Velocity
}

\author{
Enrico Bertolazzi, Francesco Biral, Mauro Da Lio, Member, IEEE and Marco Galvani
}

\begin{abstract}
Past research projects on intelligent vehicles have already led to the development of a large number of Advanced Driver Assistance Systems. The current research focus is now shifting towards integration and adaptive automation systems that share the control between driver and the machine. Artificial co-drivers can be used for this scope, as tutors to provide holistic support to the driver. However the question of the accuracy and robustness of co-driver evaluations, with respect to perception noise, becomes critical.

This work discusses the robustness to perception noise of a Curve Support function, as part of a holistic driver support system based on a co-driver concept. The main objective of the work is to respond to the following question: how accurate should the vehicle state estimation be to design a reliable system? The paper gives a general framework and preliminary results related to noise in the estimation of the vehicle velocity vector.
\end{abstract}

\section{INTRODUCTION}

Intelligent Vehicles are becoming more and more complex every day [1], [2].

One reason for complexity is the emergence of driver assistance functions of increasing sophistication, and, above all, the push for integration of many such functions into holistic driver support systems [3]-[6].

Another reason for complexity is the integrated nature itself of the aimed future systems. In facts, systems that support drivers on a holistic basis will be sort of driver companions, opening new aspects of human-machine interactions that are closely related to human-robot interaction [7]-[10].

This paper is part of the interactIVe project, which is a project of the European Framework Programme 7 tackling systematic integration of preventive and active safety functions.

In particular we focus on a function called "continuous driver support" (subproject SECONDS). This function integrates many driver support functions for a large number of scenarios; e.g., it provides proper management of speed (including green driving advices) and lane keeping/changing in relation to road geometry, curvature, obstacles, etc.

The continuous support function is intended to be a sort of tutor, which helps the driver in the appropriate moment and task, if and when necessary. It is realized by means of an artificial "co-pilot", which is smart enough to be able to

Manuscript received July 19, 2010. This work was supported in part by the European Commission under Grant FP7 246587 (interactIVe).

E. Bertolazzi, F. Biral, M. Da Lio and M. Galvani are with the Department of Mechanical and Structural Engineering at the University of Trento, via Mesiano, 77 I-38123 Povo (TN), Italy

\{enrico.bertolazzi,francesco.biral,mauro.dalio,marco.galvani\}@ing.unitn.it produce humanlike motion plans, and thus to assess whether driver behaviour is consistent with one of the possible manoeuvres.

We inherit the humanlike co-pilot idea from our previous work carried out in PReVENT [10]-[11]. However similar ideas (co-drivers/co-pilots/mentors/peers/tutors/h-metaphor) can be found also elsewhere: for example in the adaptive automation domain [12], or the rider-horse metaphor [9]. For instance a simple co-pilot, which implements some elements of the rider-horse metaphor (essentially task repartition between driver and co-driver) is being implemented in project HAVEit [5].

The structure of the co-pilot we are focusing on is based on the "sense-think-act" robotic paradigm [13]. In INSAFES project we named these three layers as perception (sense), decision (think) and action (act). The reader can refer to paper [10] for more details on our implementation and some additional literature. Of course, similar three layer structures are quite obvious and can be easily found in other Advanced Driver Assistance Systems (e.g., [36]), as well as in autonomous vehicles [14]-[15].

Unlike autonomous vehicles the co-pilot of SECONDS produces humanlike manoeuvres [11]. It does not drive the car at all, but the similarity of co-pilot manoeuvres to human ones, makes it possible to interpret driver behaviour, somewhat like there were a human tutor in the car. This is how continuous driver support is born.

In the interactIVe project, as a preliminary activity, we began to tackle the problem of robustness of the co-pilot evaluations to noise in the perception platform. One scope was to set specifications on the accuracy of the perception platform. In facts, should the sensorial system perceive a distorted reality, the thereafter decision process of the codriver would be based on an inaccurate representation of the world. A discrepancy would appear between driver and codriver manoeuvres; which is due to inaccurate perception rather than driver incorrect behaviour, thus faking the "peer co-driver" approach itself. It would be just like having a "hallucinated" tutor.

In this paper we present an analysis of a first aspect of the problem, which concerns the robustness of co-driver recommendations to errors in the sensed vehicle speed. We carry out a sensitivity analysis for a curve negotiation scenario (Fig.1), which is one of the scenarios foreseen for the interactIVe continuous support function. 


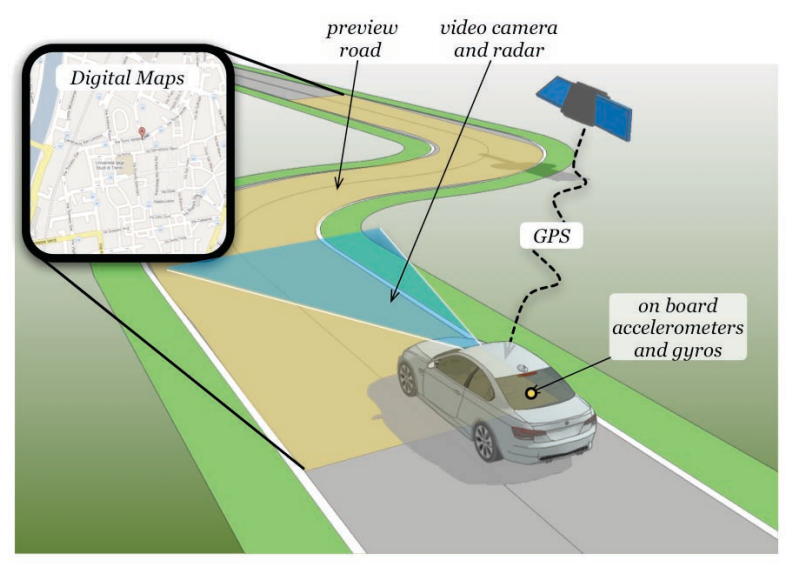

Fig. 1 Curve support system scenario and sensors

\section{SYSTEM OVERVIEW}

\section{A. InteractIVe/SECONDS System Architecture}

Fig.2 shows the system architecture of the continuous support function. Note the said three layers.

Note also the "Reference Manoeuvre" block inside the Decision layer (i.e., one of the two blocks in the Application module). The Reference manoeuvre is indeed the output of the humanlike co-driver. It is named so because the manoeuvres computed by the co-driver are used as comparison bases to interpret driver behaviour, which is carried out in the following information warning and intervention (IWI) strategies block.

The "Application" module overall implements the tutor functionality.

The types of computed reference manoeuvres change according to the vehicle-driver-environment state. For example, in a curvy road the system will evaluate, among the others, one manoeuvre to keep the vehicle in the lane and properly negotiate the curve ahead, which is the most probable driver goal. Alternative goals can also be evaluated. For example in two-lanes scenarios both in-lane and lane change alternatives [10] can be computed to assist the driver in relation to what he chooses and to what is safer.

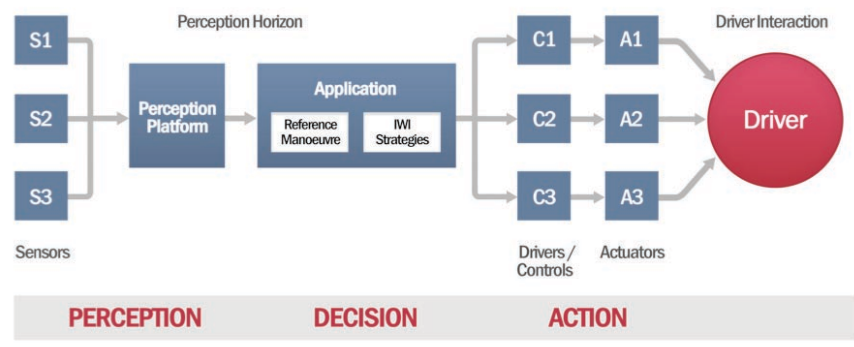

Fig.2 Application general architecture

The co-driver manoeuvres are based on a representation of the world called the Perception Horizon, which is produced by the perception layer (Fig.2). The perception horizon fuses elements like the ego vehicle state (position, velocity, etc.), the environment (road geometry and attributes from the Advanced Driver Assistance Systems Interface Specifications (ADASIS) horizon [16]), and other sensed vehicles and obstacles. It is thus clear that the quality of the representation of the world produced at the perception layer will have a profound influence on the quality of the reference manoeuvres and thus on the quality of the tutor inside the car. That is why in the first phase of the design of the continuous support function we decided to analyse the effects of errors in the perception horizon.

\section{B. The co-pilot}

Our implementation is explained in [11], [17]-[19]. We summarize here the method in order to clarify the link between errors in the perception of the world and the reference manoeuvres. Of course there are many other methods for trajectory planning, e.g., [20]-[24].

In our case the reference manoeuvres are computed by means of optimal control in the real time. This means that the vehicle motion $\boldsymbol{x}(t)$ and related driver input $\boldsymbol{u}(t)$ are chosen to minimize a goal function $\boldsymbol{J}($.) over a planning receding horizon $H$ (typically 200 meters ahead):

Minimize: $\int_{H} J(\boldsymbol{x}, \boldsymbol{u}, \boldsymbol{p}) d t$

The function $\boldsymbol{J}($.$) is the key to model humanlike manoeuvres.$ In facts human beings move smoothly [25]-[29] (i.e., they are jerk limited) and like accelerations within a predefined set, e.g., [30]-[34]. Our formulation for the optimal control criterion (1) combines the above criteria - preferred acceleration sets and jerk imitations - with quickness of motion, and is better described in [35]. We found it to produce manoeuvres that are quite similar to human ones [11].

Here we note that in the optimal control criterion (1), $\boldsymbol{p}$ is a vector of parameters that depend on the driver-vehicleenvironment state, and which are thus set by the perception horizon (Fig.2).

In addition, in the optimal control problem, the motion of the vehicle $\boldsymbol{x}(t)$ and driver control $\boldsymbol{u}(t)$ that appear in (1) are constrained by the equations of motion of the vehicle (we use a bicycle-like model, see [11] for detailed equations):

$A(x, u, p) \dot{x}=f(x, u, p)$

which again may include parameters $\boldsymbol{p}$ that are related to the driver-vehicle-environment state. Moreover the motion $\boldsymbol{x}(t)$ must satisfy initial conditions that represent the origin vehicle state (again a dependency from parameters $\boldsymbol{p}$ defined by the perception platform),

Initial conditions: $\boldsymbol{b}(\boldsymbol{x}(\boldsymbol{\theta}), \boldsymbol{p})=0$

and must comply with final conditions that represent possible driver goals (as said there may be alternative goals and multiple co-driver manoeuvres):

Final conditions: $\boldsymbol{e}(\boldsymbol{x}(H), \boldsymbol{p})=0$ 
Lastly, trajectory constraints may hold during the motion, which take the form of inequality constraints, in which we can again identify parameters that depend on the environment, vehicle and driver:

$$
g(x, u, p)<0
$$

The set of equations (1-5) is the co-driver, which, given driver-vehicle-environment parameters $\boldsymbol{p}$, returns a reference manoeuvre. As said details of our implementations and of other motion planning methods can be found in the listed papers. What is important to stress here is the link, between parameters $\boldsymbol{p}$ and the reference manoeuvres $\boldsymbol{x}(t), \boldsymbol{u}(t)$.

The IWI block (information warning and intervention strategies) will compare the co-driver reference manoeuvres $\boldsymbol{x}(t), \boldsymbol{u}(t)$ with driver behaviour (just like a tutor) and issue warnings if driver behaviour is inconsistent with any of the possible manoeuvres (IWI could also suggest the best manoeuvre among the possible ones).

Since parameters $\boldsymbol{p}$ detected by the perception horizon are affected by noise, it is important to assess the robustness of co-driver manoeuvres to such noise, and this is done here for vehicle speed errors in a curve scenario.

\section{Co-driver manoeuvres in a curve scenario}

In this section we explain how the co-driver works in a curve negotiation manoeuvre, as in Fig.3.

Let us consider a vehicle approaching a curve at a given speed $u_{0}$. Assume that the vehicle is at distance $s_{0}$ from the curve, with $s_{0}$ smaller than the planning horizon (Fig.3, point A).

Let the co-driver compute a reference manoeuvre (Fig.4) with initial conditions matching current vehicle state, and in particular speed and acceleration. In the upper charts of Fig.4 the longitudinal speed and acceleration of the reference manoeuvre are shown.

If $s_{0}$ is large enough, the reference speed may show an initial increase followed by a later reduction to negotiate the curve (Fig.4). If the distance to the curve is smaller, there may be no such possibility to accelerate before the curve (Fig.5).

The lower chart in Fig.4 shows the co-driver longitudinal jerk (jerk is the longitudinal control input $\boldsymbol{u}$ in our model). The initial value of jerk represents the input a driver should produce to follow the co-driver speed and acceleration. In general the real driver will not follow the exact co-driver plans (e.g., Fig.5). However the co-driver continues to evaluate what the driver should do. In a certain sense the jerk evaluated by the co-driver is a measurement of the discrepancy between the acceleration set by the driver and the one the co-driver would prefer.

Since we know that real drivers are likely to use a limited amount of longitudinal jerk [25]-[29], [35], [11], we can in principle detect the ultimate moment in which a real driver can no longer follow the co-driver plan, just like a tutor understands that a driver should react quicker than he usually do/can [11].

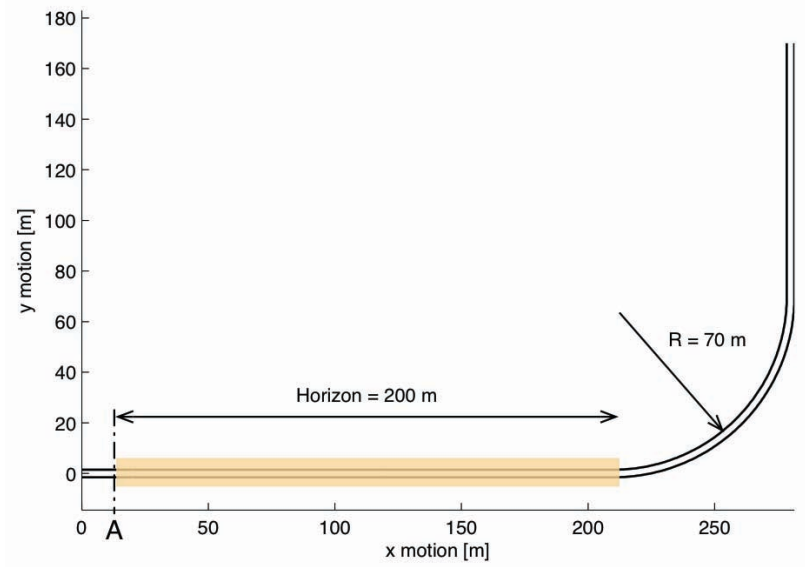

Fig. 3. Curve scenario. The shown horizon is relative to the plan at $\mathrm{s} 0=200$ $\mathrm{m}$, when the vehicle starts seeing the curve

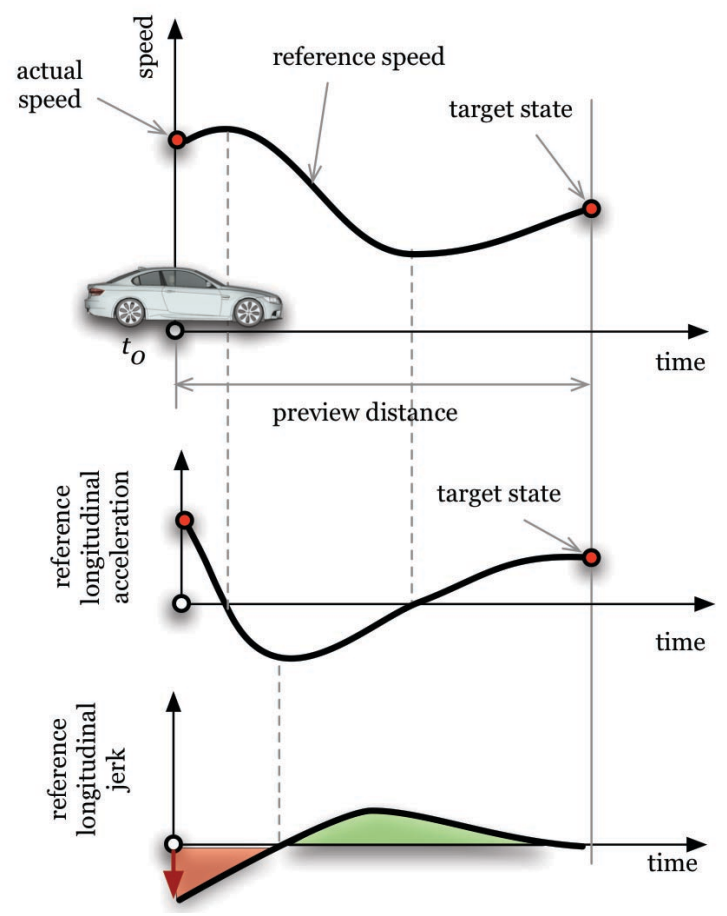

Fig. 4 Relationship between jerks and vehicle states

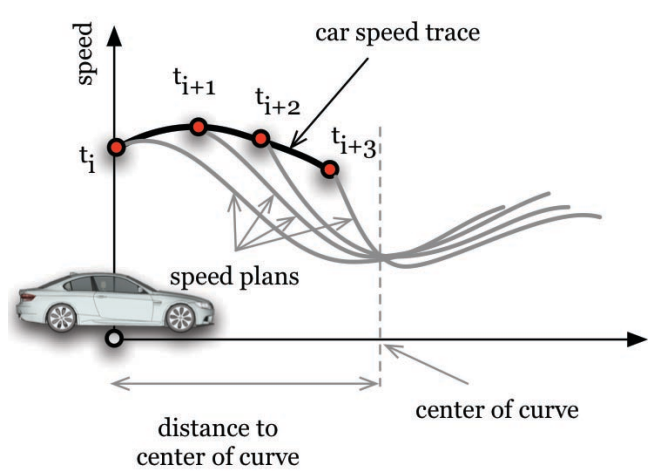

Fig. 5 Reference manoeuvres as the vehicle gets closer to the curve. 


\section{SENSITIVITY ANALYSIS}

Suppose a car moves at constant velocity towards a curve as in the scenario depicted in Fig.3. Let us compute the reference manoeuvres at various distances from the curve.

Fig. 6 plots the co-driver initial jerk as function of distance $s_{0}$ from the curve, for different velocities and zero initial acceleration. We can see that jerk is affected by the curve only if the distance $s$ is smaller than the planning horizon (A). If speed is low, e.g. $u_{0}=75 \mathrm{~km} / \mathrm{h}$, speed is safe for curve negotiation, and the system computes a positive jerk, whichever the distance. However, starting from $u 0=90$ $\mathrm{km} / \mathrm{h}$, there is a distance at which initial jerk becomes smaller than a threshold that we set here at $-1.3 \mathrm{~m} / \mathrm{s}^{3}$ (this threshold is based on values found in previous work and might change). The points where jerk falls below the threshold, are marked with circles. They are the locations where the mismatch between driver and co-driver is big enough that warnings are going to be issued. For example, if the speed were $90 \mathrm{~km} / \mathrm{h}$, the warning would be issued at few meters before the curve, but if only the speed were $95 \mathrm{~km} / \mathrm{h}$ the warning is going to be issued at about $50 \mathrm{~m}$ from the curve.
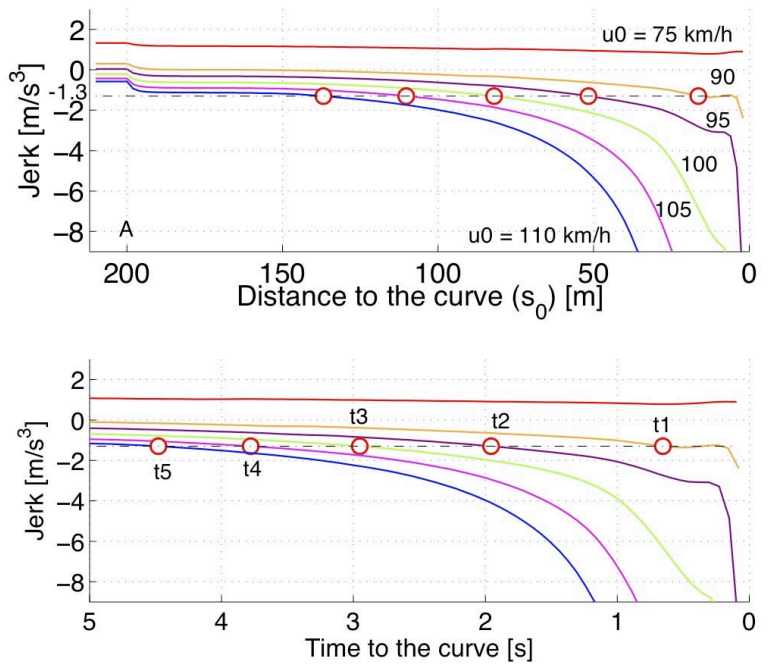

Fig. 6 Co-driver longitudinal jerks at different distances s0 to the curve (above), and at different times to the curve (below). $\mathrm{t} 1, \mathrm{t} 2, \mathrm{t} 3, \mathrm{t} 4$ and $t 5$ are the warning times to the curve at the different velocities.

The lower chart in Fig. 6 plots the jerk as a function of the "time to the curve", which is defined as $t=s_{0} / u_{0}$, i.e., the time the vehicle would need to reach the beginning of the curve if no correction were applied. So, if the initial speed is $90 \mathrm{~km} / \mathrm{h}$, the threshold is reached at $t_{1}=0.65 \mathrm{~s}$ from the curve. However, for $95 \mathrm{~km} / \mathrm{h}$ the threshold is reached $1.9 \mathrm{~s}$ before the curve: $5 \mathrm{~km} / \mathrm{h}$ of extra speed turns into $1.25 \mathrm{~s}$ anticipation of the warning. Proper timing is a critical aspect for human factors: $1 \mathrm{~s}$ delay may be critical, and $1 \mathrm{~s}$ anticipation may be annoying. For example, according to [36] a proper budget for the time error of the waning could be in the range of $+/-0.3 \mathrm{~s}$. Should the positioning subsystem of the vehicle be inaccurate, that would cause inaccurate timing of the warnings, which cannot be corrected by any post-processing at the IWI stage.
Fig. 7 plots the warning time to the curve as a function of vehicle velocity $u_{0}$ assuming that both acceleration and lateral velocity are zero. As seen, warning time is a function of vehicle velocity: $t=f\left(u_{0}\right)$. At low velocities, zero warning time means that no warning is issued.

\section{A. Sensitivity to errors in velocity estimation}

Suppose now that the velocity $u_{0}$ is affected by an estimation error $\Delta u_{0}$, i.e., $u_{0}$ is the real velocity but the system computes the reference manoeuvre with respect to a measured velocity that is $u_{0}+\Delta u_{0}$. This causes an error in warning time which is approximately $\Delta t=f^{\prime}\left(u_{0}\right) \Delta u_{0}$. If the error $\Delta u_{0}$ is assumed to be a Gaussian random variable with standard deviation $\sigma_{u}$, then the error in the warning time is also Gaussian, with standard deviation $\sigma_{t}=\left|f^{\prime}\left(u_{0}\right)\right| \sigma_{u 0}$.

Thus, we define the sensitivity coefficient of the warning time with respect to errors in longitudinal velocity as:

$\varepsilon_{t, u}=\left|f^{\prime}\left(u_{0}\right)\right|$

Fig. 8 plots the sensitivity coefficient as a function of the vehicle velocity, evaluated as the derivative of a proper interpolating function. It can be seen that at higher velocities warning times are less sensible to errors than at lower ones (the dip at about $103 \mathrm{~km} / \mathrm{h}$ is related to the fact that the planning horizon is no longer extending full way into the curve at the time of the warning).

If we set an acceptable error budget of the warning time $\sigma_{t}$, then we find that the acceptable standard deviation for the longitudinal velocity estimation error is $\sigma_{u}=\sigma_{t} / \varepsilon_{t, u}$. For example, given $3 \sigma_{t}=0.3 \mathrm{~s}, \sigma_{\mathrm{u}}$ ranges approximately between $0.1 \mathrm{~m} / \mathrm{s}$ for $90 \mathrm{~km} / \mathrm{h}$ and $0.2 \mathrm{~m} / \mathrm{s}$ for $110 \mathrm{~km} / \mathrm{h}$.

\section{B. Sensitivity to combined longitudinal-lateral velocity errors}

If both longitudinal $u(t)$ and lateral $v(t)$ velocities are considered, the warning time becomes a function of the two variables:

$t=f\left(u_{0}, v_{0}\right)$

The mathematical expectation for $\Delta t^{2}$, assuming that no correlation exists between $u$ and $v$ errors, becomes:

$E\left(\Delta t^{2}\right)=f_{u}^{\prime}(\cdot)^{2} E\left(\Delta u^{2}\right)+f_{v}^{\prime}(\cdot)^{2} E\left(\Delta v^{2}\right)$

Which shows that the error budget is, in principle, now split between $u$ and $v$.

Fig.9 shows the warning time (7) as a function of $u$ and $v$. It is clear that for high longitudinal speed (compared to safe speed) the warning time is rather insensitive to lateral speed error. This is explained by the fact that the warning occurs far from the curve, and that a small amount of lateral velocity can be corrected without affecting the trajectory in the curve. However if the forward speed is slightly greater than the safe speed the warning occur when the vehicle is close to the beginning of the curve. In this case a small 
amount of lateral velocity can facilitate or make more difficult to enter the curve, depending on the sign of the lateral speed (i.e., pointing inwards or outwards) and on the distance from the curve. That is why the contour lines of the warning time take the complex behaviour depicted in Fig.9.

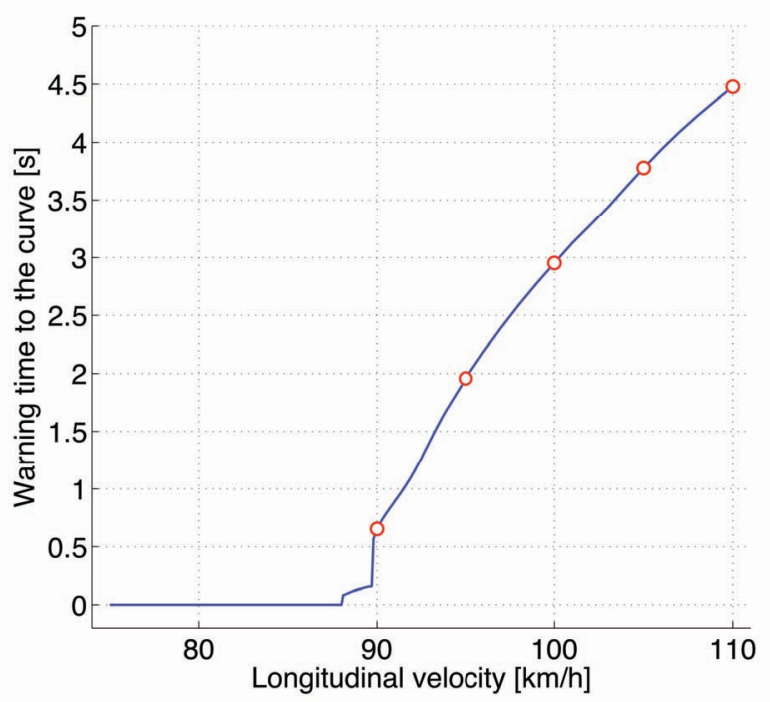

Fig. 7. Warning times to the curve.

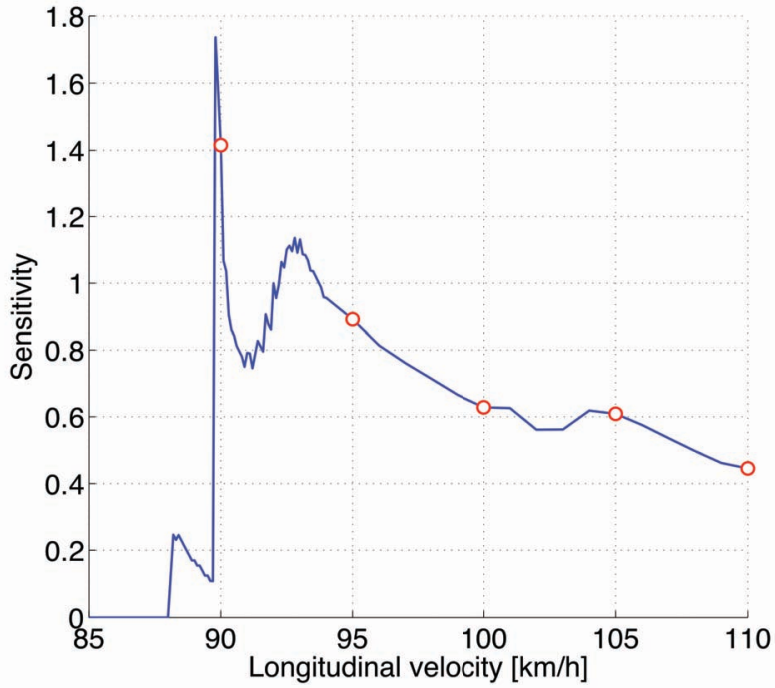

Fig.8. Sensitivity of warning times to longitudinal velocity errors $\left[\mathrm{s}^{2} / \mathrm{m}\right]$.

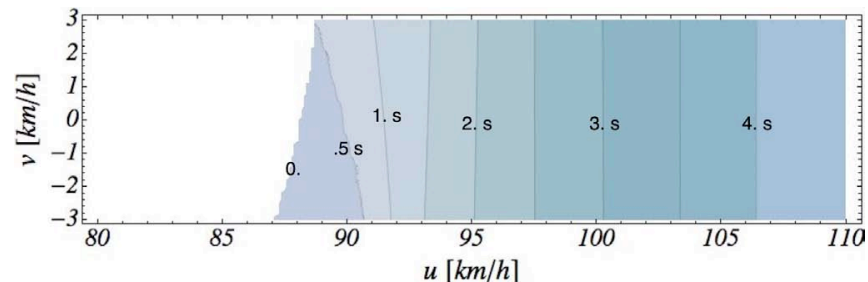

Fig.9. Warning time as a function of combined longitudinal and lateral speed.

\section{CONCLUSIONS}

This work contributes to the understanding of how perception errors propagate into a holistic driver support system based on the co-driver approach. The sensitivity of the co-driver manoeuvres is analysed with respect to errors in longitudinal and lateral velocity. Results show that in the vicinity of the curve (for speed slightly greater than the safe speed) lateral velocity interferes with the longitudinal velocity to determine the warning time. As the speed is greater, the warning occurs far from the curve and moderate lateral velocity errors have a negligible effect.

We provide also an estimation of the error budget for the velocity. However this conclusion is optimistic because drawn on the assumption that, of the many parameters $\boldsymbol{p}$ that can affect co-driver manoeuvres, only the longitudinal velocity is noisy.

In fact this is not the case. Future work will have to address the whole set of noise factors, which inevitably force to split the error budget and will lead to tighter requirements for the perception horizon accuracy.

Among noisy parameters we identify three gross classes: 1) parameters related to the vehicle state (velocity, acceleration, heading angle etc.); 2) parameters related to the description of the environment and road network (for example accuracy of curvatures, slopes and the existence of useable surfaces beyond the lane markings); 3) parameters related to driver goals and driver styles (e.g., the specific driver acceleration willingness envelope).

\section{ACKNOWLEDGEMENTS}

The authors would like to thank the European Commission for funding the interactIVe project within which this work has been carried out.

\section{REFERENCES}

[1] R. Bishop, "Intelligent vehicle R\&D: "A review and contrast of programs worldwide and emerging trends," Ann. Telecommunications, vol. 60, no. 3-4, pp. 228-263, 2005.

[2] J. Piao, M. McDonald, "Advanced Driver Assistance Systems from Autonomous to Cooperative Approach," Transport Reviews, vol. 28, no. 5, pp. 659-684, 2008.

[3] [Online]. Available: http://world.honda.com/HDTV/ASV/ASV-3auto/index.html

[4] [Online]. Available: http://www.prevent-ip.org/

[5] [Online]. Available: http://www.haveit-eu.org/

[6] R. Harrington, A. Lam, E. Nodine, J. Ference, W. G. Najm, "Integrated Vehicle-Based Safety Systems (IVBSS) - Light Vehicle On-Road," Test Report, sponsored by National Highway Traffic Safety Administration, Washington D.C., August 2008, DOT HS 811 020 (http://www.nhtsa.dot.gov, section vehicle safety research, crash avoidance publications).

[7] T. Inagaki, "Smart collaboration between humans and machines based on mutual understanding," Annual Reviews in Control, vol.32, pp. 253-261, 2008.

[8] A. Heide, K. Henning, "The "cognitive car"': A roadmap for research issues in the automotive sector," Annual Reviews in Control, vol. 30, pp. 197-203, 2006.

[9] F. Flemisch, C. Adams, S. Conway et al., "The H-Metaphor as a guideline for vehicle automation and interaction," NASA, TM-2003212672, Dec 2003.

[10] A. Amditis, E. Bertolazzi, M. Bimpas, F. Biral, M. Da Lio, L. Danielsson, H. Lind, A. Saroldi, A. Sjogren, "A Holistic Approach to the Integration of Safety Applications: the INSAFES Subproject within the European Framework Program 6 Integrating Project PReVENT," in IEEE Trans. on Intelligent Transportation Systems, 
special issue on ITS and Road Safety, to be published, Digital Object Identifier 10.1109/TITS.2009.2036736.

[11] E. Bertolazzi, F. Biral, M. Da Lio, A. Saroldi, and F. Tango, "Supporting drivers in keeping safe speed and safe distance: The SASPENCE subproject within the European framework program 6 integrating project PReVENT," IEEE Trans. Intell. Transp. Syst., special issue on ITS and Road Safety, to be published, Digital Object Identifier 10.1109/TITS.2009.2035925.

[12] D. A. Norman, "The Design of Future Things". New York: Basic Books. November, 2007.

[13] A. C. Ronald, "Behavior-Based Robotics", MIT Press , (1998), ISBN 0-262-01165-4.

[14] S. Thrun, M. Montemerlo, et al., "Stanley: The Robot that Won the DARPA Grand Challenge", Journal of Field Robotics 23(9), 661-692 (2006)

[15] M. Montemerlo, J. Becker, S. Bhat, H. Dahlkamp, D. Dolgov, S. Ettinger, D. Haehnel, "Junior: The Stanford Entry in the Urban Challenge,” J. Field Robotics, vol. 25, no. 9, pp. 569-597, 2008.

[16] C. Ress, D. Balzer, A. Bracht, S, Durekovic, J, Löwenau, ADASIS PROTOCOL FOR ADVANCED IN-VEHICLE APPLICATIONS, http://www.ertico.com/download/ADASIS_Forum_documents/ADAS ISv2\%20ITS\%20NY\%20Paper \%20Final v4.pdf

[17] E. Bertolazzi, F. Biral, and M. Da Lio, "Symbolic-numeric indirect method for solving optimal control problems for large multibody systems - The time-optimal racing vehicle example," Multibody Syst. Dyn., vol. 13, no. 2, pp. 233-252, Mar. 2005.

[18] E. Bertolazzi, F. Biral, and M. Da Lio, "Symbolic-numeric efficient so- lution of optimal control problems for multibody systems," J. Comput. Appl. Math., vol. 185, no. 2, pp. 404-421, Jan. 2006.

[19] E. Bertolazzi, F. Biral, and M. Da Lio, "Real-time motion planning for multibody systems," Multibody Syst. Dyn., vol. 17, no. 2/3, pp. 119139, Apr. 2007.

[20] J. Bruce, M. Veloso, "Real-time randomized path planning for robot navigation,". In: Proceedings of the IEEE-RSJ Intelligent Robots and Systems, 2002, pp. 2383-2388.

[21] S.M. LaValle, J.J. Kuffner, "Randomized kinodynamic planning," Int. J. Robot. Res., vol.20-5, 2001, pp. 378-400

[22] W.T. Cerven, F. Bullo, V.L. Coverstone, "Vehicle motion planning with time-varying constraints," J. Guid. Control Dyn., vol. 27-3, 2004, pp. 506-509.

[23] O. von Stryk, R. Bulirsch, "Direct and indirect methods for trajectory optimization," Ann. Oper. Res., vol. 37-1-4, 1992, pp. 357-373 (1992).

[24] J.T. Betts, "Survey of numerical methods for trajectory optimization," J. Guid. Control Dyn., vol. 21- 2, 1998, pp. 193-207.

[25] Breteler, M., Meulenbroek, R., \& Gielen, S. (2002) An evaluation of the minimum-jerk and minimum torque-change principles at the path, trajectory, and movement-cost levels. Motor Control 6, 69-83.

[26] Arechavaleta, G., Laumond, J., Hicheur, H., \& Berthoz, A. (2008) An optimality principle governing human walking. Ieee Transactions On Robotics 24, 5-14.

[27] Abdel-Malek, K., Mi, Z., Yang, J., \& Nebel, K. (2006) Optimizationbased trajectory planning of the human upper body. Robotica 24, 683696.

[28] Pham, Q., Hicheur, H., Arechavaleta, G., Laumond, J., \& Berthoz, A. (2007) The formation of trajectories during goal-oriented locomotion in humans. II. A maximum smoothness model. European Journal of Neuroscience 26, 2391-2403.

[29] D Liu, E. Todorov, Evidence for the Flexible Sensorimotor Strategies Predicted by Optimal Feedback Control, Journal of Neuroscience, 2007 • 27(35):9354-9368

[30] R.S., Rice. "Measuring car-driver interaction with the g-g diagram," SAE Paper 730018, 1973.

[31] Y Hisaoka, M. Yamamoto, A. Okada, "Closed-loop analysis of vehicle behavior during braking in a turn", JSAE Review, vol. 20, pp. 537-542, Apr 1999.

[32] W. Bartlett, L. L. C. Oren Masory, B. Wright, "Driver Abilities in Closed Course Testing”, SAE Technical Paper Series, 2000-01-0179.

[33] S. T., Godley, "A driving simulator investigation of perceptual countermeasures to speeding", Ph.D. Thesis, Department of Psychology Monash University, 1999.

[34] R.J.V. Bertin, ,A. Berthoz, "Visuo-vestibular interaction in the reconstruction of travelled trajector- ies", http://cogprints.org/, 2002.
[35] F. Biral, M. Da Lio, and E. Bertolazzi, "Combining safety margin and user preferences into a driving criterion for optimal control-based computation of reference maneuvers for an ADAS of the next generation," in Proc. IEEE Intell. Veh. Symp., Las Vegas, NV, 2005, pp. 36-41. DOI: 10.1109/IVS.2005.1505074.

[36] D. Pomerleau, T. Jochem, C. Thorpe, P. Batavia, D. Pape, J. Hadden Nancy McMillan, N. Brown, J. Everson, "Run-Off-Road Collision Avoidance Using IVHS Countermeasures," DOT HS 809 170, Final Report, p. 88, December 1999. 